\title{
Surgical Treatment for Ulnar Nerve Entrapment at the Elbow
}

\author{
Shunji ASAMOTO, Dieter-Karsten BÖKER, and Andreas JÖDICKE \\ Department of Neurosurgery, Justus-Liebig University of Gießen, Gießen, Germany
}

\begin{abstract}
The outcomes of 81 operations were assessed for the treatment of ulnar nerve entrapment at the elbow performed on 55 males (bilateral operations in one) and 25 females during the period from January 1995 to December 2000. Before operation, neurophysiological examination was performed in all patients. Simple ulnar nerve decompression or anterior transposition of the ulnar nerve (subcutaneous or intramuscular) was performed with or without the operating microscope. Nine patients were lost to follow up. The outcome was excellent or good in 63 of 72 cases, no change in eight cases, and poor in one case. The outcomes of procedures performed with the operating microscope tended to be superior.
\end{abstract}

Key words: ulnar nerve entrapment, elbow, surgical treatment

\section{Introduction}

Ulnar nerve entrapment (UNE) is the second most common nerve entrapment syndrome of the upper extremity. ${ }^{8-10,27,29,31,32,34-36,39,42)}$ The initial treatment of acute and subacute neuropathy is conservative. Rest and avoidance of pressure on the nerve may suffice, but if symptoms persist, splint immobilization of the elbow is warranted..$^{12,33,40)}$ Surgery is usually necessary to treat chronic neuropathy associated with muscle weakness or neuropathy that does not respond to conservative measures. Several surgical and non-surgical treatments have been proposed for the treatment of this neuropathy. ${ }^{6,7,13,17)}$ Deciding on the most effective procedure can be difficult, given the excellent results claimed. ${ }^{1,2,14,18)}$ Despite the high frequency of UNE, there is no clear consensus on the indications for surgery or the most appropriate surgical technique. ${ }^{14-16,19,20,23-25,30,41)}$ Operative management includes simple decompression (or "decompression in situ"); medial epicondylectomy, either alone or in combination with simple decompression; and anterior transposition.

We present a retrospective study of the outcomes of surgical treatment for UNE.

\section{Patients and Methods}

\section{Patient population}

The outcomes of 81 operations for UNE performed in 80 patients, 55 males aged 21 to 80 years (mean 53.7 years) and 25 females aged 28 to 73 years (mean 52.3 years), including one male who underwent bilateral operations during the period from January 1995 to December 2000 in our department were analyzed. No cases were associated with trauma. The follow-up period ranged from 1 to 24 months (mean 2.2 months).

\section{Diagnosis}

After enrollment in the study and prior to surgery, patients completed a detailed preoperative questionnaire regarding the presence of pain or numbness, the location of the symptoms, and work-related issues. Preoperative electromyography (EMG) and nerve conduction velocity studies were performed bilaterally in all patients using standard techniques. Electrodiagnostic studies are useful to confirm the diagnosis of cubital tunnel syndrome, and to determine the severity of the disease, to localize the area of compression in some cases, and to exclude other sites of compression. Motor and sensory conduction velocities were determined in a standardized fashion, evaluating the ulnar nerve across the elbow

Received July 2, 2004; Accepted December 3, 2004

Author's present address: S. Asamoto, M.D., Department of Neurosurgery, Tokyo Mita Hospital, International University of Health and Welfare, Tokyo, Japan. 
and from below the elbow to wrist. ${ }^{3,5,8,21,25,35)}$

\section{Indication for surgical intervention}

In our hospital, the decision for surgical treatment is made by a neurosurgeon in cooperation with a neurologist. Treatment of UNE was divided into non-operative (conservative) and operative strategies. Generally, patients reporting only intermittent paresthesia and normal clinical findings were managed conservatively. Non-operative management included educating the patient to avoid pressure on the elbow, to avoid prolonged elbow flexion as during reading or telephoning, to modify the work environment by optimizing the height of the work surface, and to pad surfaces on which the elbow and the forearm rest. Patients with fixed sensory loss, pain, weakness, or significant denervation on EMG were considered for surgical treatment.

\section{Surgical technique}

Surgical intervention was performed under general anesthesia by well-trained neurosurgeons in our institute. Patients were positioned supine, and the externally rotated arm was placed on the arm-table with the elbow flexed and the forearm in the supine position. An omega skin incision of about $10 \mathrm{~cm}$ in length was made about the medial epicondyle. Two different operative procedures were used: simple ulnar nerve decompression without epicondylectomy, and anterior transposition of the ulnar nerve (subcutaneous or intramuscular) with or without use of a microscope (Table 1). The choice of procedure was made at the surgeon's discretion, but simple decompression was performed only in patients who had mild symptoms. Except for simple decompression, anterior transposition of the ulnar nerve was performed as a rule. However, submuscular anterior transposition was selected in some patients with severely tensed ulnar nerve due to cubitus valgus over $30^{\circ}$ or those engaging in work using the inside of the elbow or those receiving re-operation. In addition, the decision to use a microscope was made at random by the operator. Because few patients had relatively mild preoperative neurological findings, there were differences in the number of patients in the simple decompression and anterior transposition groups.

\section{Results}

Table 2 summarizes the results of surgery for UNE. Neurologists and neurosurgeons evaluated the postoperative neurological findings. Nine patients were lost to follow up. Outcome was excellent or good in 63 of 72 cases, without changes in signs or symptoms in eight cases and poor in one case. Since the mode of operation was selected under the conditions mentioned above, no comparison was made for different modes of operation. However, a comparative study was performed of individual modes of operation with or without use of a microscope. As a result, it was found that the outcomes of surgery with or without use of the operating microscope also showed no significant difference, but the outcomes with microscopic technique tended to be superior. All patients without improvement of neurological findings had severe preoperative symptoms. Therefore, cases of poor outcome may be related to preoperative changes in the perineural vessels. Of course, no complication was observed in the perioperative findings and procedures in cases of poor outcome. Though only a speculation, it is estimated that patients with poor outcome were in a hypersensitive state, so easily suffered damage

Table 1 Operative procedures for ulnar nerve entrapment

\begin{tabular}{lcc}
\hline & $\begin{array}{c}\text { With } \\
\text { microscope }\end{array}$ & $\begin{array}{c}\text { Without } \\
\text { microscope }\end{array}$ \\
\hline $\begin{array}{l}\text { Simple decompression } \\
\text { Anterior transposition }\end{array}$ & 5 & 8 \\
$\quad$ subcutaneous & 25 & 33 \\
intramuscular & 3 & 7 \\
\hline
\end{tabular}

Table 2 Results of surgery for ulnar nerve entrapment

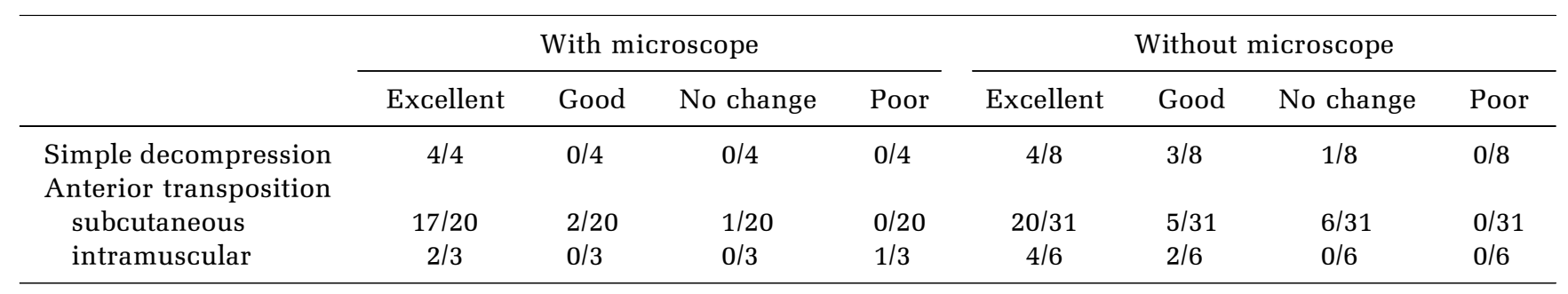

Nine patients lost to follow up. Total of 72 cases evaluated in 71 patients. 
caused by mobilizing nerves or perineural vessels due to severe perioperative neurological findings.

\section{Discussion}

Operative procedures can be divided into two groups: decompression (including medial epicondylectomy) with transposition, and decompression without transposition. Decompression without transposition is also referred to as decompression in situ or "simple decompression." Transposing the nerve has two main advantages. The first is that the nerve removed from an unsuitable bed and repositioned to one that is less scarred. ${ }^{3-5)}$ This is necessary for many types of lesions, that are proximal to or within the epicondylar groove..$^{1,18,19,21)}$ The second advantage is that by transposing the nerve into a new pathway volar to the axis of elbow motion, the nerve is effectively lengthened, which decreases tension with elbow flexion. There are three methods for transposition: subcutaneous transposition, intramuscular transposition, and submuscular transposition. ${ }^{4,5,22,28,30,37)}$ We did not perform submuscular transposition in our series.

Subcutaneous transposition is the most commonly used method of ulnar nerve transposition because it is not technically difficult, has a high success rate, and is the least complicated. ${ }^{3,38)}$ Subcutaneous transposition is an effective procedure, particularly in the elderly and patients who have a thick layer of adipose tissue. The disadvantages of subcutaneous transposition are risk of failure to decompress the nerve at the most distal site (cubital tunnel of the flexor carpi ulnaris muscle), and the nerve remains vulnerable to repeated trauma, particularly in thin, active individuals. ${ }^{7,19)}$

Submuscular transposition can potentially cause more scarring than the other surgical procedures. The advantages of submuscular transposition are that potential sites for nerve compression are definitely explored and released, and the nerve lies in an unscarred anatomic plane not subject to traction forces. ${ }^{7,24,27)}$ However, submuscular transposition is usually contraindicated if there is scarring of the joint capsule or distortion of the joint due to arthritis or a malunited fracture.

Intramuscular transposition is the most controversial of the three methods of ulnar nerve transposition..$^{22,31,33,38)}$ In addition, the outcome differed distinctly according to the neurological severity before operation, which is an issue separate from the selection of surgical procedure. The outcomes of surgical manipulations for UNE can only be compared if a randomized prospective study is performed in populations with almost equivalent preoperative conditions, but such conditions are difficult to obtain. ${ }^{10,16,18,23,42)}$ Various outcomes of different surgical treatments for this neuropathy have been described, so it is difficult to assess which surgical procedure is the best for this disease. ${ }^{1,2,4-6,8,26,38,42)}$

Microsurgical technique allows the perineural vessels to be preserved in decompression and, to a lesser degree, in anterior transposition procedures, so the use of the surgical microscope seems to be important. In this study, microsurgical treatment tended to improve clinical outcome in patients with UNE. These results should be reconfirmed in a prospective controlled study with randomized use of microsurgical technique. No other examination of various surgical procedures with or without the operating microscope in the treatment of this neuropathy is available.

Both simple decompression and surgical manipulation to improve the articular mobile region are clearly required in some patients with UNE, although we experienced no such case in our series.

In this series, no decision could be made with confidence concerning which mode of operation is the best for treatment of UNE. Some authors reported that simple decompression and anterior transposition produced equal outcomes. ${ }^{37}$ As mentioned previously, however, potential difference by difference modes of operation seems to be more clearly depicted if a study is performed by grouping the patient population more specifically and systematically according to the preoperative neurological findings or to the mobilizing conditions of elbow joint, and then by selecting a mode of operation in a random fashion. However, we think such a study may require a large number of cases or extensive cooperation with several institutes. Unfortunately, no distinct significant difference was obtained, probably due to the small number of patients enrolled in this series. We believe that the outcome tends to be distinctly good when a microscopic procedure is used in different modes of operation, although no significant difference was observed statistically.

Our department works closely with the department of orthopedics, thus allowing prompt consultation whenever such cases arise. ${ }^{7,11,43)}$ In Japan, only a few neurosurgeons are committed to surgery of the peripheral nerves. Neurosurgeons should reconsider the use of the surgical microscopes for peripheral nerve surgery to potentially improve the outcome of their patients. 


\section{Acknowledgments}

The authors wish to acknowledge Dr. Katsunori Inagaki, Department of Orthopedics, Showa University School of Medicine, Tokyo, for secretarial support and assistance.

This work was supported by a grant from the Japanese Neurosurgical Foundation (2000 Sep.).

\section{References}

1) Amako $M$, Nemoto $K$, Kawaguchi $M$, Kato N, Arino $\mathrm{H}$, Fujikawa K: Comparison between partial and minimal medial epicondylectomy combined with decompression for the treatment of cubital tunnel syndrome. J Hand Surg [Am] 25: 1043-1050, 2000

2) Arle JE, Zager EL: Surgical treatment of common entrapment neuropathies in the upper limbs. Muscle Nerve 23: 1160-1174, 2000 (review)

3) Artico M, Pastore FS, Nucci F, Giuffre R: 290 surgical procedures for ulnar nerve entrapment at the elbow: physiopathology, clinical experience and results. Acta Neurochir (Wien) 142: 303-308, 2000

4) Bartels RH: History of the surgical treatment of ulnar nerve compression at the elbow. Neurosurgery 49: 391-400, 2001 (review)

5) Bartels RH, Menovsky T, Van Overbeeke JJ, Verhagen WI: Surgical management of ulnar nerve compression at the elbow: an analysis of the literature. J Neurosurg 89: 722-727, 1998 (review)

6) Bimmler D, Meyer VE: Surgical treatment of the ulnar nerve entrapment neuropathy: submuscular anterior transposition or simple decompression of the ulnar nerve? Long-term results in 79 cases. Ann Chir Main Memb Super 15: 148-157, 1996

7) Black BT, Barron OA, Townsend PF, Glickel SZ, Eaton RG: Stabilized subcutaneous ulnar nerve transposition with immediate range of motion. Long-term follow-up. J Bone Joint Surg Am 82-A: 1544-1551, 2000

8) Bozentka DJ: Cubital tunnel syndrome pathophysiology. Clin Orthop (351): 90-94, 1998 (review)

9) Bradshaw DY, Shefner JM: Ulnar neuropathy at the elbow. Neurol Clin 17: 447-461, v-vi, 1999 (review)

10) Campbell WW: Ulnar neuropathy at the elbow. Muscle Nerve 23: 450-452, 2000 (review)

11) Capuano L, Craig N, Ashcroft GP, Maffulli N: Distraction lengthening of the radius for radial longitudinal instability after distal radio-ulnar subluxation and excision of the radial head: a case report. Scand J Plast Reconstr Surg Hand Surg 35: 331-335, 2001

12) Filippi $R$, Charalampaki $P$, Reisch $R$, Koch $D$, Grunert P: Recurrent cubital tunnel syndrome. Etiology and treatment. Minim Invasive Neurosurg 44: 197-201, 2001

13) Gellman H, Campion DS: Modified in situ decompression of the ulnar nerve at the elbow. Hand Clin 12: 405-410, 1996
14) Geutjens GG, Langstaff RJ, Smith NJ, Jefferson D, Howell CJ, Barton NJ: Medial epicondylectomy or ulnar-nerve transposition for ulnar neuropathy at the elbow? J Bone Joint Surg Br 78: 777-779, 1996

15) Gobel F, Musgrave DS, Vardakas DG, Vogt MT, Sotereanos DG: Minimal medial epicondylectomy and decompression for cubital tunnel syndrome. Clin Orthop (393): 228-236, 2001

16) Grana W: Medial epicondylitis and cubital tunnel syndrome in the throwing athlete. Clin Sports Med 20: 541-548, 2001 (review)

17) Green JR Jr, Rayan GM: The cubital tunnel: anatomic, histologic, and biomechanical study. J Shoulder Elbow Surg 8: 466-470, 1999

18) Grewal R, Varitimidis SE, Vardakas DG, Fu FH, Sotereanos DG: Ulnar nerve elongation and excursion in the cubital tunnel after decompression and anterior transposition. J Hand Surg [Br] 25: 457-460, 2000

19) Jackson LC, Hotchkiss RN: Cubital tunnel surgery. Complications and treatment of failures. Hand Clin 12: 449-456, 1996

20) Kleinman WB: Cubital tunnel syndrome: anterior transposition as a logical approach to complete nerve decompression. J Hand Surg [Am] 24: 886-897, 1999

21) Kohut GN, Della Santa DR, Chamay A: [Ulnar nerve compression syndrome of the elbow. Analysis of 50 operated cases]. Ann Chir Main Memb Super 15: 138-147, 1996 (Fre, with Eng abstract)

22) Leone J, Bhandari $M$, Thoma A: Anterior intramuscular transposition with ulnar nerve decompression at the elbow. Clin Orthop (387): 132-139, 2001

23) Lowe JB 3rd, Novak CB, Mackinnon SE: Current approach to cubital tunnel syndrome. Neurosurg Clin N Am 12: 267-284, 2001

24) Lundborg G: Surgical treatment for ulnar nerve entrapment at the elbow. J Hand Surg [Br] 17: 245-247, 1992

25) Merlevede K, Theys P, van Hees J: Diagnosis of ulnar neuropathy: a new approach. Muscle Nerve 23: 478-481, 2000

26) Nakazumi Y, Hamasaki M: Electrophysiological studies and physical examinations in entrapment neuropathy: sensory and motor functions compensation for the central nervous system in cases with peripheral nerve damage. Electromyogr Clin Neurophysiol 41: 345-348, 2001

27) Nathan PA: Surgical treatment of ulnar nerve entrapment at the elbow. J Hand Surg [Br] 18: 133, 1993

28) Osterman AL, Davis CA: Subcutaneous transposition of the ulnar nerve for treatment of cubital tunnel syndrome. Hand Clin 12: 421-433, 1996

29) Padua L, Aprile I, Mazza O, Padua R, Pietracci E, Caliandro P, Pauri F, D'Amico P, Tonali P: Neurophysiological classification of ulnar entrapment across the elbow. Neurol Sci 22: 11-16, 2001

30) Paternostro-Sluga T, Ciovika R, Turkof E, ZaunerDungl A, Posch M, Fialka-Moser V: Short segment stimulation of the anterior transposed ulnar nerve at the elbow. Arch Phys Med Rehabil 82: 1171-1175, 
2001

31) Pellieux S, Fouquet B, Lasfargues G: [Ulnar nerve tunnel syndrome of the elbow and an occupational disorder. Analysis of socio-professional and physical parameters]. Ann Readapt Med Phys 44: 213-220, 2001 (Fre, with Eng abstract)

32) Posner MA: Compressive ulnar neuropathies at the elbow: I. Etiology and diagnosis. J Am Acad Orthop Surg 6: 282-288, 1998 (review)

33) Posner MA: Compressive ulnar neuropathies at the elbow: II. treatment. J Am Acad Orthop Surg 6: 289-297, 1998 (review)

34) Richardson JK, Green DF, Jamieson SC, Valentin FC: Gender, body mass and age as risk factors for ulnar mononeuropathy at the elbow. Muscle Nerve 24: 551-554, 2001

35) San Martin S, Bueno C, Montes C, Diaz-Calavia E, Teijeira JM, Lopez-Reneo R: [The most significative parameters for the diagnosis of focal neuropathy of the cubital nerve in the elbow]. Rev Neurol 31: 720-723, 2000 (Spa, wit Eng abstract)

36) Sharma RR, Pawar SJ, Delmendo A, Mahapatra AK: Symptomatic epineural ganglion cyst of the ulnar nerve in the cubital tunnel: a case report and brief review of the literature. J Clin Neurosci 7: 542-543, 2000 (review)

37) Steiner HH, von Haken MS, Steiner-Milz HG: Entrapment neuropathy at the cubital tunnel: simple decompression is the method of choice. Acta Neurochir (Wien) 138: 308-313, 1996

38) Tada H, Hirayama T, Katsuki M, Habaguchi T: Long term results using a modified King's method for cubital tunnel syndrome. Clin Orthop (336): 107-110, 1997

39) Tomaino MM, Brach PJ, Vansickle DP: The rationale for and efficacy of surgical intervention for electrodiagnostic-negative cubital tunnel syndrome. J Hand Surg [Am] 26: 1077-1081, 2001

40) Valls Canals J, Povedano M, Montero J, Pradas J: [Electrophysiological evaluation of level compression in elbow ulnar neuropathy]. Neurologia 14: 389-392, 1999 (Spa, with Eng abstract)

41) Vardakas DG, Varitimidis SE, Sotereanos DG: Findings of exploration of a vein-wrapped ulnar nerve: report of a case. J Hand Surg [Am] 26: 60-63, 2001

42) Wang FC, Crielaard JM: [Entrapment neuropathies in sports medicine]. Rev Med Liege 56: 382-390, 2001 (Fre, with Eng abstract)

43) Wright TW, Glowczewskie F Jr, Cowin D, Wheeler DL: Ulnar nerve excursion and strain at the elbow and wrist associated with upper extremity motion. J Hand Surg [Am] 26: 655-662, 2001

Address reprint requests to: S. Asamoto, M.D., Department of Neurosurgery, Tokyo Mita Hospital, International University of Health and Welfare, 1-4-3 Mita, Minato-ku, Tokyo 108-8329, Japan.

e-mail: spine-ns@sb.dcns.ne.jp

\section{Commentary}

Surgical treatment of ulnar nerve entrapment at the elbow is indeed a common problem. As pointed out in the article, this type of peripheral nerve decompression operation is secondary only to median nerve decompressions at the carpal tunnel. The authors of this manuscript have done a good job reviewing 81 cases. That is over a five-year period, which means they are doing 16-20 cases per year. Our own experience is only about 10 per year over the last 30 years. The authors have done a better job in the follow-up than we have.

We stratify patients similarly to the authors. We prefer to do the simpler decompression through a rather short incision coming from the mid point between the medial epicondyle of the humerus and the olecranon of the ulnar bone. For a simpler decompression we simply start an incision next to the elbow and carry it down the forearm 3 to $4 \mathrm{~cm}$ over the course of the nerve. In many of these cases we find a very thick fibrous band of the cubical tunnel of the flexor carpii ulnaris muscle. Basically that fascia has to be opened, and you can go subcutaneous with basic surgical scissors to make sure the nerve is free below that. We use this as the first operation in a majority of patients. We will do that under local anesthesia, with maybe some very mild IV sedation.

In patients who have considerably more severe symptoms, and who have had previous surgeries, then we agree that the subcutaneous transposition needs to be done. Our surgical technique is very similar to the authors but a more extensive exposure may have to be done. The goal is really to achieve a fresh bed for the nerve. We found the disadvantage of that procedure is when the patient straightens out their arm completely. That still caused stretching of the nerve, so often we will splint the patient after this procedure in a half-cast where the arm remains slightly flexed by 30 degrees. They are put in this simple posterior splint, and sleep that way for about a month, which cuts down on the scarring and trauma to the nerve. Then they learn not to extend their elbow all the way and avoid stretching symptomatology. We feel the more extensive operation is best as a salvage procedure.

In all honesty, I do not use the microscope. I have my headlight and my loupes and I have gotten used to that. Maybe I should do as the authors point out; however, the authors do point out it is hard to show a significant difference in using a more expensive technology.

All of us need to remember that even in expert hands, $87 \%$ of these patients do very well, and certainly not a $100 \%$. The challenge can become even 
greater in those who have any hint of diabetes and other peripheral neuropathies.

Thomas B. DuCKER, M.D., F.A.C.S. Department of Neurosurgery Johns Hopkins University Annapolis, Maryland, U.S.A.

The authors presented beautifully their excellent surgical results of $87.5 \%$ improvement rate in a series of 72 patients with ulnar nerve entrapment of the elbow. According to my past experience, poor surgical results have always been associated with longer duration of symptoms, and dense adhesive bands and tendinous structures entrapping the certain segment of the ulnar nerve somewhere near the cubital tunnel, causing irreversible changes. The authors, however, claim poor outcome cases were related to what they call "hypersensitive state" which is rather difficult to understand in terms of pathomechanism. Readers will be able to learn more if the authors could provide us high core evidence of poor surgical outcome, particularly by focusing on duration of symptoms and operative findings. The authors should be congratulated, however, for bringing to attention and discussing the less mentioned topics of peripheral nerve surgery in Japan.

Hiromichi Hosoda, M.D. Department of Neurosurgery Chigasaki Tokushukai Medical Center Chigasaki, Kanagawa, Japan 